\title{
On the power boundedness of certain Volterra operator pencils
}

\author{
by \\ DAshdondog TSEDEnbayar (Warszawa and Ulan-Bator)
}

Abstract. Let $V$ be the classical Volterra operator on $L^{2}(0,1)$, and let $z$ be a complex number. We prove that $I-z V$ is power bounded if and only if $\operatorname{Re} z \geq 0$ and $\operatorname{Im} z=0$, while $I-z V^{2}$ is power bounded if and only if $z=0$. The first result yields

$$
\left\|(I-V)^{n}-(I-V)^{n+1}\right\|=O\left(n^{-1 / 2}\right) \quad \text { as } n \rightarrow \infty,
$$

an improvement of $[\mathrm{Py}]$. We also study some other related operator pencils.

1. Preliminaries. We say that an operator $A$ is power-bounded if $\sup _{n \geq 0}\left\|A^{n}\right\|<\infty$. We denote by $V$ the classical Volterra operator

$$
(V f)(x)=\int_{0}^{x} f(s) d s, \quad 0<x<1, \quad \text { on } L^{p}(0,1), 1 \leq p \leq \infty .
$$

We recall the well-known formula

$$
\left(V^{n} f\right)(x)=\int_{0}^{x} \frac{(x-s)^{n-1}}{(n-1) !} f(s) d s \quad \text { for } n \in \mathbb{N} .
$$

A generalization of this formula is the definition of the Riemann-Liouville integral operator of any fractional order $\alpha>0$,

$$
\left(J^{\alpha} f\right)(x)=\frac{1}{\Gamma(\alpha)} \int_{0}^{x}(x-s)^{\alpha-1} f(s) d s
$$

( $\Gamma$ is the Euler gamma function) on $L^{p}(0,1), 1 \leq p \leq \infty$. In particular, $V=J^{1}$.

Recall that the Ritt condition for the resolvent $R(\lambda, A)=(A-\lambda I)^{-1}$ of a bounded linear operator $A$ on a Banach space is

$$
\|R(\lambda, A)\| \leq \frac{\text { const }}{|\lambda-1|}, \quad|\lambda|>1,
$$

2000 Mathematics Subject Classification: Primary 47A10.

Key words and phrases: Volterra operator, power-bounded operator, resolvent. 
which is equivalent to a geometric condition much stronger than the power boundedness of $A$ [NaZe], [Ne2]. If the operator $A$ is merely power-bounded, then the weaker Kreiss condition

$$
\|R(\lambda, A)\| \leq \frac{\text { const }}{|\lambda|-1}, \quad|\lambda|>1,
$$

holds, but not conversely in general.

2. Introduction. In 1997, Allan [Al] recorded the observation made by T. V. Pedersen that $I-V$ is similar to $(I+V)^{-1}$, namely

$$
S^{-1}(I-V) S=(I+V)^{-1}
$$

where $(S f)(t)=e^{t} f(t), f \in L^{p}(0,1), 1 \leq p \leq \infty$. By [Ha, Problem 150], we know that $\left\|(I+V)^{-1}\right\|=1$ on $L^{2}(0,1)$. Hence $I-V$ is a power-bounded operator on $L^{2}(0,1)$.

In 1987, Pytlik [Py], basing on an upper estimate for the Fejér expression for Laguerre polynomials (see [Sz, p. 198]), proved

$$
\left\|(I-V)^{n}-(I-V)^{n+1}\right\|=O\left(n^{-1 / 4}\right)
$$

as $n \rightarrow+\infty$ on $L^{2}(0,1)$. The same argument gives the same result also on $L^{1}(0,1)$, in which case it is sharp [ToZe]. By this method, one is unable to distinguish the delicate properties of the $L^{p}$-norms. We shall show, by an algebraic argument, the power boundedness of $I-t V$ for $t>0$, on $L^{2}(0,1)$, which will improve Pytlik's estimate to $O\left(n^{-1 / 2}\right)$. Our method, however, does not apply to $L^{1}(0,1)$, because $I-V$ is not power-bounded there (see [Hi, p. 247]), and (2) actually cannot be improved on $L^{1}(0,1)$ as mentioned above [ToZe]. We also study some other related operator pencils. The details of some calculations as well as alternative proofs of some cases are given in $[\mathrm{Ts}]$.

\section{The results}

Proposition 1. Let $A$ and $B$ be two commuting power-bounded operators on a Banach space, and $0 \leq t \leq 1$. Then the convex combination $t A+(1-t) B$ is a power-bounded operator.

Proof. By the binomial formula,

$$
\begin{aligned}
& \left\|(t A+(1-t) B)^{n}\right\| \leq \sum_{k=0}^{n}\left(\begin{array}{l}
n \\
k
\end{array}\right) t^{k}\left\|A^{k}\right\|(1-t)^{n-k}\left\|B^{n-k}\right\| \\
& \leq \mathrm{const} \sum_{k=0}^{n}\left(\begin{array}{l}
n \\
k
\end{array}\right) t^{k}(1-t)^{n-k}=\operatorname{const}(t+(1-t))^{n}=\mathrm{const} .
\end{aligned}
$$


THEOREM 1. The operator $I-z V$ is power-bounded on $L^{2}(0,1)$ if and only if $\operatorname{Re} z \geq 0$ and $\operatorname{Im} z=0$.

Proof. (If) It follows from Proposition 1 and the power boundedness of $I-V$ (explained above) that $I-t V=(1-t) I+t(I-V)$ is power-bounded for $0 \leq t \leq 1$ on $L^{2}(0,1)$.

Let $m$ be a natural number. Note the following extension of formula (1):

$$
S^{-1}(I-m V) S=(I-(m-1) V)(I+V)^{-1}
$$

where $(S f)(t)=e^{t} f(t), f \in L^{p}(0,1)$. We shall verify it by induction. If $m=1$, we have (1). Suppose that (3) holds for some $m$. Then

$$
\begin{aligned}
S^{-1}(I-(m+ & 1) V) S \\
& =I-S^{-1}(m V) S-S^{-1} V S \\
& =(I-(m-1) V)(I+V)^{-1}+(I+V)^{-1}-I \\
& =(I-(m-1) V)(I+V)^{-1}+(I+V)^{-1}-(I+V)(I+V)^{-1} \\
& =(I-(m-1) V)(I+V)^{-1}+(I-(I+V))(I+V)^{-1} \\
& =(I-m V)(I+V)^{-1} .
\end{aligned}
$$

This proves (3) and yields the power boundedness of $I-m V$ for all $m \in \mathbb{N}$.

Then the convex combination $(1-t)(I-m V)+t(I-(m+1) V)=$ $I-(m+t) V$ is power-bounded for $0 \leq t \leq 1$ and $m \in \mathbb{N}$.

(Only if) We shall show that the operator $I-z V$ does not satisfy the Kreiss condition on $L^{2}(0,1)$ for $\operatorname{Im} z \neq 0$. Thus $I-z V$ is not power-bounded on this space for those $z$. Indeed, using the well-known formula for the resolvent of $V$ (see e.g. [Ne1, p. 27]), we obtain

$$
(R(\lambda, I-z V) f)(x)=-\frac{f(x)}{\lambda-1}+\frac{z}{(\lambda-1)^{2}} \int_{0}^{x} e^{-(x-s) z /(\lambda-1)} f(s) d s, \quad \lambda \neq 1 .
$$

We have

$$
\begin{array}{ll}
\limsup _{n \rightarrow \infty}(|1+i / n|-1)\left\|R(1+i / n, I-z V) e^{i n \cdot}\right\|=\infty & \text { for } \operatorname{Im} z<0, \\
\limsup _{n \rightarrow \infty}(|1-i / n|-1)\left\|R(1-i / n, I-z V) e^{i n \cdot}\right\|=\infty & \text { for } \operatorname{Im} z>0 .
\end{array}
$$

Of course, $I-z V$ is not power-bounded for $\operatorname{Re} z<0$ and $\operatorname{Im} z=0$, because for $f \equiv 1$, we have $\lim _{\sup _{n \rightarrow \infty}}\left\|(I-z V)^{n} 1\right\|=\infty$.

Corollary 1. On $L^{2}(0,1)$, we have

$$
\left\|(I-V)^{n}-(I-V)^{n+1}\right\|=O\left(n^{-1 / 2}\right) \quad \text { as } n \rightarrow \infty .
$$


Proof. Set $L=I-\mu V$ for $\mu>1$, which is power-bounded by Theorem 1 . Then $L_{\omega}=(1-\omega) I+\omega L=(1-\omega) I+\omega(I-\mu V)=I-\omega \mu V$ is power-bounded for $0<\omega<1$ by Proposition 1. Now, Nevanlinna's theorem [Ne1, Theorem 4.5.3] yields

$$
\limsup _{n \rightarrow \infty} n^{1 / 2}\left\|L_{\omega}^{n}\left(L_{\omega}-I\right)\right\| \leq \operatorname{const}\left(\frac{\omega}{2 \pi(1-\omega)}\right)^{1 / 2} .
$$

So, for $\omega=1 / \mu$ we get the claim.

Remark 1. Corollary 1 does not follow from Nevanlinna's paper [Ne2, p. 121] because his resolvent assumption (1.35) is not satisfied for any positive $\alpha<1$.

REMARK 2. Alternatively, one can also use [FoWe, Lemma 2.1] instead of [Ne1, Theorem 4.5.3]; observe that the proof in [FoWe, Lemma 2.1] works also for power-bounded commuting pairs, or use [BoDu, Theorem 4.1].

REMARK 3. It would be interesting to know if the above estimate $O\left(n^{-1 / 2}\right)$ is already sharp, and if it extends, together with Theorem 1, to $L^{p}(0,1), 1<p<\infty$. The above proof of Theorem 1 extends to these spaces as soon as we know that $I-V$ is power-bounded there. Perhaps the Riesz-Thorin convexity theorem [BeSh, p. 196] could be applied.

REMARK 4. It has been pointed out by Yuri Tomilov that Corollary 1 also follows from [Sa] and (1), by using [FoWe] as above. However, this approach does not seem to give Theorem 1 . On the other hand, our Theorem 1 yields the corresponding information about the power boundedness of the Sarason operator pencil.

Remark 5. Consider the matrix

$$
\mathbb{A}=\left(\begin{array}{ll}
0 & 1 \\
0 & 0
\end{array}\right)
$$

Then $I-z \mathbb{A}, z \in \mathbb{C}$, is power-bounded if and only if $z=0$.

TheOREM 2. The operator $I-z V, z \in \mathbb{C}$, is power-bounded on $L^{1}(0,1)$ if and only if $z=0$.

Proof. We consider the following three cases:

Case $t<0$. The operator $I-t V$ is not power-bounded on $L^{1}(0,1)$ for $t<0$ since as before, from the binomial formula it is clear that

$$
\limsup _{n \rightarrow \infty}\left\|(I-t V)^{n} 1\right\|=\infty .
$$


Case $t>0$. As in $[\mathrm{Py}$, p. 292] we can write

$$
\begin{array}{r}
\left((I-t V)^{n} f\right)(x)-\left((I-t V)^{n+1} f\right)(x)=\left(t V(I-t V)^{n} f\right)(x) \\
=t\left(\sum_{k=0}^{n}\left(\begin{array}{l}
n \\
k
\end{array}\right)(-1)^{k} t^{k} V^{k+1} f\right)(x)=t \int_{0}^{x} \sum_{k=0}^{n}\left(\begin{array}{l}
n \\
k
\end{array}\right)(-1)^{k} t^{k} \frac{(x-s)^{k}}{k !} f(s) d s \\
=t \int_{0}^{x} L_{n}^{(0)}(t(x-s)) f(s) d s
\end{array}
$$

where

$$
L_{n}^{(0)}(x)=\sum_{k=0}^{n}\left(\begin{array}{l}
n \\
k
\end{array}\right)(-1)^{k} \frac{x^{k}}{k !}, \quad n \geq 1,
$$

are the Laguerre polynomials with parameter 0 . By summing these formulas and using [Sz, p. 102, formula (5.1.13)], we get

$$
\left((I-t V)^{n+1} f\right)(x)=f(x)-t \int_{0}^{x} L_{n}^{(1)}(t(x-s)) f(s) d s
$$

where $L_{n}^{(1)}(x)$ are the Laguerre polynomials with parameter 1 .

Using the classical estimates for Laguerre polynomials [Sz, p. 177 and $198]$ and the formula for the norm of an integral operator on $L^{1}(0,1)$ given in [ToZe, Lemma 4.5], we deduce as in [ToZe, Example 4.6] that

$$
\limsup _{n \rightarrow \infty}\left\|(I-t V)^{n}\right\|=\infty \text {. }
$$

Case $z \in \mathbb{C} \backslash \mathbb{R}$. We show that the operator $I-z V$ does not satisfy the Kreiss condition on $L^{1}(0,1)$ for $\operatorname{Im} z \neq 0$. Thus $I-z V$ is not power-bounded on $L^{1}(0,1)$ for those $z$. Indeed, on $L^{1}(0,1)$, we have

$$
\begin{array}{ll}
\limsup _{n \rightarrow \infty}(|1+i / n|-1)\left\|R(1+i / n, I-z V) e^{i n \cdot}\right\|=\infty & \text { for } \operatorname{Im} z<0, \\
\limsup _{n \rightarrow \infty}(|1-i / n|-1)\left\|R(1-i / n, I-z V) e^{i n \cdot}\right\|=\infty & \text { for } \operatorname{Im} z>0 .
\end{array}
$$

REMARK 6. By duality, the same characterization holds on $L^{\infty}(0,1)$.

Proposition 2. Let $\sigma(Q)=\{0\}$. If $I-Q$ satisfies the Ritt condition, then so does $I-t Q$ for $t>0$.

Proof. We can write

$$
\begin{aligned}
R(\lambda, I-t Q) & =(I-t Q-\lambda I)^{-1}=\frac{1}{t}\left(\frac{1-\lambda}{t} I-Q\right)^{-1} \\
& =\frac{1}{t}\left[(I-Q)-I+\frac{1-\lambda}{t} I\right]^{-1} \\
& =\frac{1}{t}\left[I-Q-\left(1-\frac{1-\lambda}{t}\right) I\right]^{-1}
\end{aligned}
$$


Whenever $|t-1+\lambda|>t$, i.e. $|\lambda-(1-t)|>t$, which certainly holds for $\operatorname{Re} \lambda>1$, we have

$$
\|R(\lambda, I-t Q)\| \leq \frac{1}{t} \frac{\text { const }}{\left|\frac{t-1+\lambda}{t}-1\right|}=\frac{\text { const }}{|\lambda-1|},
$$

and this yields the Ritt condition by [NaZe, Lemma, p. 146] because $\sigma(Q)$ $=\{0\}$.

REMARK 7. The operator $I-J^{\alpha}$ satisfies the Ritt condition for $0<$ $\alpha<1$ on $L^{p}(0,1), 1 \leq p \leq \infty$, by [Ly2, p. 137], hence $I-t J^{\alpha}$ satisfies the Ritt condition for all $t>0$ on $L^{p}(0,1), 1 \leq p \leq \infty$, by Proposition 2 . Hence these operators are power-bounded by [Ly1, Theorem 1, p. 154] or [NaZe, Theorem, p. 147].

This observation does not seem to follow by the method used above in the case $\alpha=1$, because there is no analogy of (1) and (3) for $\alpha \neq 1$.

We know from Theorem 1 that $I-t V$ is power-bounded on $L^{2}(0,1)$, while $I+t V$ is not for $t>0$ (for $t=1$ the latter also follows from the Gelfand Theorem $[\mathrm{Ge}])$. This leads to the natural question whether the product $(I-t V)(I+t V)=I-t^{2} V^{2}$ is power-bounded. The answer is negative.

TheOREM 3. The operator $I-z V^{2}, z \in \mathbb{C}$, is power-bounded on $L^{p}(0,1)$, $1 \leq p \leq \infty$, if and only if $z=0$.

Proof. We consider the following three cases:

Case $t<0$. The operator $I-t V^{2}$ is not power-bounded on $L^{p}(0,1)$, $1 \leq p \leq \infty$, for $t<0$ because, as before, from the binomial formula it is clear that

$$
\limsup _{n \rightarrow \infty}\left\|\left(I-t V^{2}\right)^{n} 1\right\|=\infty .
$$

Case $t>0$. The resolvent formula for $V^{2}$ is

$$
\begin{aligned}
& \left(R\left(\lambda, I-V^{2}\right) f\right)(x) \\
& \quad=-\frac{f(x)}{\lambda-1}+\frac{1}{(\lambda-1)^{3 / 2}} \int_{0}^{x} \sinh \frac{x-s}{(\lambda-1)^{1 / 2}} f(s) d s \quad \text { for } \lambda \neq 1
\end{aligned}
$$

(see [Hi, p. 260] or [Ne1, p. 130]). Therefore the resolvent formula for $I-t V^{2}$ is

$$
\begin{aligned}
\left(R\left(\lambda, I-t V^{2}\right) f\right)(x) & =\frac{1}{t}\left(R\left(1-\frac{1-\lambda}{t}, I-V^{2}\right) f\right)(x) \\
& =-\frac{f(x)}{\lambda-1}+\frac{t^{1 / 2}}{(\lambda-1)^{3 / 2}} \int_{0}^{x} \sinh \frac{(x-s) t^{1 / 2}}{(\lambda-1)^{1 / 2}} f(s) d s .
\end{aligned}
$$


We choose $f \equiv 1$. Then

$$
\begin{aligned}
\left(R\left(\lambda, I-t V^{2}\right) 1\right)(x) & =-\frac{1}{\lambda-1}+\frac{t^{1 / 2}}{(\lambda-1)^{3 / 2}} \int_{0}^{x} \sinh \frac{(x-s) t^{1 / 2}}{(\lambda-1)^{1 / 2}} d s \\
& =-\frac{2}{\lambda-1}+\frac{1}{\lambda-1} \cosh \frac{t^{1 / 2} x}{(\lambda-1)^{1 / 2}} .
\end{aligned}
$$

We note that

$$
\begin{aligned}
& \int_{0}^{x} \sinh \frac{(x-s) t^{1 / 2}}{(\lambda-1)^{1 / 2}} d s \\
& \quad=-\frac{(\lambda-1)^{1 / 2}}{t^{1 / 2}}+\frac{1}{2} \frac{(\lambda-1)^{1 / 2}}{t^{1 / 2}}\left[e^{t^{1 / 2} x /(\lambda-1)^{1 / 2}}+e^{-t^{1 / 2} x /(\lambda-1)^{1 / 2}}\right] .
\end{aligned}
$$

Hence, for $\lambda_{n}=1+1 / n$, we get

$$
\left(R\left(\lambda_{n}, I-t V^{2}\right) 1\right)(x)=-2 n+n \cosh \sqrt{n t} x=n(\cosh \sqrt{n t} x-2),
$$

and an easy calculation shows that

$$
\limsup _{n \rightarrow \infty}\left(\left|\lambda_{n}\right|-1\right)\left\|\left(R\left(\lambda_{n}, I-t V^{2}\right) 1\right)(x)\right\|=\infty .
$$

Therefore, $R\left(\lambda, I-t V^{2}\right)$ does not satisfy the Kreiss condition for $t>0$.

Case $z \in \mathbb{C} \backslash \mathbb{R}$. We show that the operator $I-z V^{2}$ does not satisfy the Kreiss condition on $L^{p}(0,1), 1 \leq p \leq \infty$, for $\operatorname{Im} z \neq 0$. Indeed, we can write $z=(\alpha+i \beta)^{2}$ with $\alpha, \beta \in \mathbb{R}$, where $\alpha \neq 0$. In the resolvent formula for $I-z V^{2}$,

$$
\left(R\left(\lambda, I-z V^{2}\right) f\right)(x)=-\frac{f(x)}{\lambda-1}+\frac{z^{1 / 2}}{(\lambda-1)^{3 / 2}} \int_{0}^{x} \sinh \frac{(x-s) z^{1 / 2}}{(\lambda-1)^{1 / 2}} f(s) d s
$$

we set $\lambda_{n}=1+1 / n^{2}$. Then

$$
\begin{aligned}
\left(R \left(1+1 / n^{2}, I\right.\right. & \left.\left.-z^{2} V^{2}\right) e^{i n \cdot}\right)(x) \\
& =-n^{2} e^{i n x}+n^{3}(\alpha+i \beta) \int_{0}^{x} \sinh [n(x-s)(\alpha+i \beta)] e^{i n s} d s .
\end{aligned}
$$

We note that

$$
\begin{aligned}
\int_{0}^{x} \sinh [n(x-s)(\alpha+i \beta)] e^{i n s} d s= & -\frac{1}{2} \frac{e^{i n x}}{n(\alpha+i(\beta-1))}+\frac{e^{n(\alpha+i \beta) x}}{2 n(\alpha+i(\beta-1))} \\
& -\frac{1}{2} \frac{e^{i n x}}{n(\alpha+i(\beta+1))}+\frac{e^{-n(\alpha+i \beta) x}}{2 n(\alpha+i(\beta+1))} .
\end{aligned}
$$

We get

$$
\limsup _{n \rightarrow \infty} \frac{1}{n^{2}}\left\|\left(R\left(\lambda_{n}, I-z V^{2}\right) e^{i n \cdot}\right)(x)\right\|=\infty .
$$

Therefore, $R\left(\lambda, I-z V^{2}\right)$ does not satisfy the Kreiss condition. 
Acknowledgments. I am grateful to Professors Yuri Tomilov and Jaroslav Zemánek for helpful discussions during the work on this paper.

\section{References}

[Al] G. R. Allan, Power-bounded elements and radical Banach algebras, in: Linear Operators, J. Janas, F. H. Szafraniec and J. Zemánek (eds.), Banach Center Publ. 38, Inst. Math., Polish Acad. Sci., 1997, 9-16.

[BeSh] C. Bennett and R. Sharpley, Interpolation of Operators, Academic Press, New York, 1988.

[BoDu] F. F. Bonsall and J. Duncan, Complete Normed Algebras, Springer, Berlin, 1973.

[FoWe] S. R. Foguel and B. Weiss, On convex power series of a conservative Markov operator, Proc. Amer. Math. Soc. 38 (1973), 325-330.

[Ge] I. Gelfand, Zur Theorie der Charaktere der Abelschen topologischen Gruppen, Mat. Sb. 9 (1941), 49-50.

[Ha] P. R. Halmos, A Hilbert Space Problem Book, Van Nostrand, Princeton, 1967.

[Hi] E. Hille, Remarks on ergodic theorems, Trans. Amer. Math. Soc. 57 (1945), 246-269.

[Ly1] Yu. Lyubich, Spectral localization, power boundedness and invariant subspaces under Ritt's type condition, Studia Math. 134 (1999), 153-167.

[Ly2] - The single point spectrum operators satisfying Ritt's resolvent condition, ibid. 145 (2001), 135-142.

[NaZe] B. Nagy and J. Zemánek, A resolvent condition implying power boundedness, ibid. 134 (1999), 143-151.

[Ne1] O. Nevanlinna, Convergence of Iterations for Linear Equations, Birkhäuser, Basel, 1993.

[Ne2] -, Resolvent conditions and powers of operators, Studia Math. 145 (2001), 113134.

[Py] T. Pytlik, Analytic semigroups in Banach algebras, Colloq. Math. 51 (1987), 287-294.

[Sa] D. Sarason, A remark on the Volterra operator, J. Math. Anal. Appl. 12 (1965), $244-246$.

[Sz] G. Szegö, Orthogonal Polynomials, Colloq. Publ. 23, Amer. Math. Soc., 1939.

[ToZe] Yu. Tomilov and J. Zemánek, A new way of constructing examples in operator ergodic theory, Math. Proc. Cambridge Philos. Soc., to appear.

[Ts] D. Tsedenbayar, Some properties of the Volterra and Cesàro operators, dissertation, Inst. Math., Polish Acad. Sci., Warszawa, 2002.

Institute of Mathematics

Polish Academy of Sciences

Śniadeckich 8

P.O. Box 21

00-956 Warszawa, Poland

E-mail: tsdnbr@impan.gov.pl
Department of Mathematics Mongolian Teacher's University Ulan-Bator, Mongolia 